\title{
Mitigated NSAID-induced apoptotic and autophagic cell death with Smad7 overexpression
}

\author{
Ho-Jae Lee, ${ }^{1, *}$ Jong Min Park² and Ki Baik Hahm²,3 \\ 'Department of Biochemistry, Gachon University School of Medicine, 7-45, Songdo-dong, Yeonsu-ku, Incheon 406-840, Korea \\ ${ }^{2} \mathrm{CHA}$ University Cancer Prevention Research Center, CHA Bio Complex, 335, Pangyo-ro, Bundang-gu, Seongnam 463-400, Korea \\ ${ }^{3} \mathrm{CHA}$ University Bundang Medical Center, 59, Yatap-ro, Bundang-gu, Seongnam 463-712, Korea
}

(Received 11 August, 2016; Accepted 7 September, 2016; Published online 17 December, 2016)

Non-steroidal anti-inflammatory drugs damaged gastrointestinal mucosa in cyclooxygenase-dependent and -independent pathway, among which apopototic or autophagic cell death in gastrointestinal cells might be one of key cytotoxic mechanisms responsible for NSAID-induced damages. Therefore, alleviating this cell death after NSAIDs can be a rescuing strategy. In this study, we explored the role of Smad7 on NSAID-induced cytotoxicity in gastric epithelial cells. Using RGM1 cells, we have compared biological changes between mock-transfected and Smad7-overexpressed cells. As results, significantly decreased cytotoxicity accompanied with decreased levels of cleaved caspase-3 and poly (ADP-ribose) polymerase, Bax, and autophagic vesicles concurrent with decreased expressions of autophagy protein 5 and microtubuleassociated protein light chain 3B-II were noted in Smad7overexpressed cells with indomethacin administration compared to mock-transfected cells. Contrast to mitigated apoptotic execution, anti-apoptotic Bcl-2 and Beclin-1 were significantly increased in Smad7-overexpressed cells compared to mock-transfected cells. Smad7 siRNA significantly reversed these protective actions of Smad7 against indomethacin, in which p38 mitogen-activated protein kinase was significantly intervened. Furthermore, indomethacin-induced Smad7 degradation through ubiquitinproteasome pathway was relevant to increased cytotoxicity, while chloroquine as autophagy inhibitor significantly attenuated indomethacin-induced cytotoxicity through Smad7 preservation via repressed ubiquitination. Conclusively, either genetic overexpression or pharmacological induction of Smad7 significantly attenuated indomethacin-induced gastric cell damages.

Key Words: NSAID, gastrointestinal damages, Smad7, apoptosis, autophagy

$\mathrm{N}$ on-steroidal anti-inflammatory drugs (NSAIDs) have been widely used, with indications extending from pain, inflammation to cardiovascular diseases and anti-cancer purpose in spite of their notorious adverse effects including abdominal pain, diarrhea, dyspepsia due to ulcer, upper gastrointestinal (GI) bleedings and bowel perforation. ${ }^{(1,2)}$ Since the primary mode of action of NSAIDs is the inhibition of prostaglandin (PG) synthesis via the inhibition of cyclooxygenase- 1 (COX-1) and COX-2, ${ }^{(3)}$ indiscernible decreases of gastroprotective prostaglandin $\mathrm{E}_{2}\left(\mathrm{PGE}_{2}\right)$ had been regarded as core contributing factor for these adverse effects, the mechanisms of NSAID-induced cytotoxicity occurred as systemic outcome of COX-dependent mechanisms.

However, as representative COX-independent mechanisms responsible to these GI adverse effects, recent molecular exploration showed that apoptosis mediated by reactive oxygen species (ROS) and endoplasmic reticulum (ER) stress has been put forwarded. ${ }^{(4,5)}$ Since cell death is a phenomenon occurring next to ER stress followed by autophagic cell death, extensive cytotoxicity can be a key arsenal responsible for NSAID-induced GI damages. However, little information is available about bad liaison between NSAID-induced gastric damages and transforming growth factor$\beta$ (TGF- $\beta$ ) signaling pathway. TGF- $\beta$ signals had been pivotally implicated in either inflammation or cytotoxicity, the liaison between NSAID and TGF- $\beta$ signal should be investigated to find the exit for rescue of GI tract. NSAID also had been tried for cancer preventive purposes, for instance, familial adenomatous polyposis and Barrett's esophagus, in which aggressive cancers are more addicted to autophagy for survival. In non-cancer cells, autophagy being also implicated to be or not to be, ${ }^{(6,7)}$ after which we put hypothesis that Smad7 as an inhibitory regulator in TGF- $\beta$ signaling pathway can be friend or foe as far as NSAIDs are concerned.

In spite of intense efforts to develop novel therapeutics like GI-safer NSAIDs, still dependence on coxibs or combination with acid suppressants is the only straitened answer next to avoidance or reduced use of NSAIDs. Therefore, elucidating underlying hitblow mechanism implicated in NSAID-induced gastric damages can yield aspiration for rescuing from gastric damages. Supported with previous publications that NSAID can induce ER stress and apoptosis ${ }^{(5,8)}$ ER stress is closely connected with autophagy activation, ${ }^{(9,10)}$ autophagy could regulate the fate of apoptosis, ${ }^{(11,12)}$ in the current study, we have explored the contribution of Smad7 on NSAID-induced cytotoxicity.

\section{Materials and Methods}

Reagents. Smad7, PARP-1/2, and $\beta$-actin from Santa Cruz Biotechnology (Santa Cruz, CA), cleaved caspase-3, LC3B, ATG5, phospho-ERK and phospho-p38 from Cell Signaling Technology (Beverly, MA), and cleaved LC3B from ABGENT (San Diego, CA). All other chemicals and reagents were purchased from Sigma-Aldrich (St. Louis, MO) unless otherwise specified.

Cell culture and cytotoxicity assay. The rat gastric mucosal cells, RGM1, were kindly given by Prof. Hirofumi Matsui (University of Tsukuba, Japan) and were maintained at $37^{\circ} \mathrm{C}$ in a humidified atmosphere containing 5\% $\mathrm{CO}_{2}$. RGM1 cells were cultured in Dulbecco's modified Eagle's medium containing 10\% (v/v) fetal bovine serum, $100 \mathrm{U} / \mathrm{ml}$ penicillin, $100 \mu \mathrm{g} / \mathrm{ml}$ streptomycin. RGM1 cells were plated and incubated for $24 \mathrm{~h}$ after which media was changed with fresh one containing indomethacin or chloroquine (CQ). Cell cytotoxicity was measured by MTT [3(4,5-dimethylthiazol-2-yl)-2,5-diphenyltetrazolium bromide] assay.

Generation of Smad7-expressing RGM1 cells. The RGM1 cells stably expressing Smad7 were generated by using pLPCX

*To whom correspondence should be addressed.

E-mail: hojlee@gachon.ac.kr (HJ Lee), hahmkb@cha.ac.kr (KB Hahm) 
retroviral vector. Flag-tagged Smad7 was inserted into pLPCX and viruses were produced according to the manufacturer's protocol (Cell Biolabs Inc., San Diego, CA). After infection, $1 \mu \mathrm{g} / \mathrm{ml}$ of puromycin was treated for selection of cells expressing Smad7. Cells containing empty pLPCX vector (RGM1-mock) were used as a control.

Western blotting and immunoprecipitation. Treated cells were washed twice with PBS and then lysed in ice-cold cell lysis buffer (Cell Signaling Technology) containing $1 \mathrm{mM}$ PMSF. Proteins in lysates were separated by SDS-PAGE followed by electro-transfer to polyvinylidene difluoride (PVDF) membranes and probed with polyclonal or monoclonal antisera, followed by horseradish peroxidase-conjugated anti-rabbit, anti-mouse IgG and visualized by chemiluminescence, according to the manufacturer's instructions (iNtRON Biotechnology, Seongnam, Korea). For immunoprecipitation, the cell lysates were incubated with the appropriated antibody for $1 \mathrm{~h}$, followed by incubation with Protein A Excellose-binding bead (Bioprogen Co., Daejeon, Korea) for $1 \mathrm{~h}$ at $4^{\circ} \mathrm{C}$. Beads were washed three times with the buffer used for cell solubilization. Immune complexes were then eluted by boiling for $5 \mathrm{~min}$ in $2 \mathrm{X}$ Tris-Glycine SDS Sample Buffer (Invitrogen, Carlsbad, CA), and then extracts were analyzed by immunoblotting as described above.

Annexin V apoptosis assay. Annexin V-FITC apoptosis kit was purchased from BD Biosciences (San Diego, CA), and performed according to manufacturer's instructions. Briefly, after treatment, the media was collected for including death cells and adherent cells were trypsinized for $5 \mathrm{~min}$ and then suspended with previously collected media. After washed, the cells were suspended with $1 \mathrm{x}$ binding buffer, adjusted to final concentration of $1.0 \times 10^{6} / \mathrm{ml}$. One hundred $\mu$ l of cell-binding buffer solution was mixed with $4 \mu \mathrm{l}$ of anti-Annexin V-FITC and propidium iodide, then solution was incubated $15 \mathrm{~min}$ at room temperature in the dark. Reactions were terminated with insertion of $400 \mu \mathrm{l}$ of binding buffer, then analysed by FACS Calibur flow cytometer (BD Biosciences).

Analysis of GFP-LC3 dots. To detect translocation of GFPLC3 from the cytosolic fraction to autophagic vacuoles, Smad7expressed RGM1 cells transfected with GFP-LC3 were grown in a Lab-Tek II chamber slide with cover glass (Nalge Nunc International, Rochester, NY) for $24 \mathrm{~h}$, after which cells were treated with indomethacin. Autofluorescence GFP-LC3 (excitation $488 \mathrm{~nm}$, emission $515 \mathrm{~nm}$ ) was observed under an LSM 700 confocal microscope (Carl Zeiss, Jena, Germany).

RNA interference. The expression of Smad7 was abolished using target-specific small interference RNA (siRNA) molecules purchased from Bioneer (Daejeon, Korea). Smad7 siRNA was transfected into RGM1 cells using Lipofectamine reagent (Invitrogen, Carlsbad, CA) according to the manufacturer's instructions.

RNA Isolation and RT-PCR. Total RNA was isolated from cells using TRIzol (Invitrogen) according to the manufacturer's protocol. Reverse transcription was performed with $1 \mu \mathrm{g}$ of pure RNA using M-MLV reverse transcriptase (Promega, Madison, WI) according to the manufacturer's instructions. The synthesized cDNA was amplified by PCR using specific primers. PCR products were visualized by electrophoresis on agarose gels with RedSafe (iNtRON Biotechnology, Korea) staining and analyzed using an Image Quant LAS 4000 image analyzer (GE Healthcare Life Sciences, Piscataway, NJ).

Statistical analysis. The data are presented as means \pm SD. The Tukey test or the Student's $t$ for unpaired results was used to evaluate differences between more than three groups or between two groups, respectively. Differences were considered to be significant for values of $p<0.05$.

\section{Results}

Smad7 mitigated indomethacin-induced apoptotic and autophagic cell death in RGM1 cells. To investigate the effect of Smad7 in indomethacin-induced gastric epithelial cell injury, we generated RGM1 non-transformed gastric mucosal cells stably expressing Flag-tagged Smad7. Smad7 expression, as compared to parental and mock-transfected RGM1 cells, was verified by Western blotting (Fig. 1A). When RGM1 cells were exposed to different concentrations of indomethacin, 250 and $500 \mu \mathrm{M}$, for $24 \mathrm{~h}$, their viabilities significantly decreased in a dose-dependent manner in mock-transfected cells $(p<0.05)$. However, the overexpression of Smad7 in RGM1 cells significantly decreased the indomethacin-induced cytotoxicity compared to mock-transfected cells $(p<0.001$, Fig. 1B). The effect of Smad7 overexpression in gastric epithelial cells was then determined by Western blot analysis to examine whether indomethacin-induced cytotoxicity was associated with apoptosis (Fig. 1C) or autophagy (Fig. 1D). Caspase- 3 and Bax activation, and resulting cleavage of poly (ADP-ribose) polymerase (PARP), are known as key events during the induction of apoptosis. As shown in Fig. 1C, treatment of indomethacin at $500 \mu \mathrm{M}$ for $24 \mathrm{~h}$ induced clearly increased levels of the cleaved forms of caspase-3, PARP and proapoptotic Bcl-2 family protein, Bax. Compared with those of mocktransfected RGM-1 cells after indomethacin, these representative markers of apoptosis were significantly reduced in Smad7overexpressed RGM1 cells (Fig. 1C), signifying that indomethacin treatment was associated with cytotoxicity in RGM1 cells via apoptotic cell death, while Smad7 mitigated these indomethacininduced apoptosis. Next, in order to see whether indomethacin induced apoptosis occurred via autophagy, we examined the levels of autophagy markers, autophagy protein 5 (ATG5), microtubule-associated protein light chain 3B-II (LC3B-II) and Beclin-1. Upon autophagy activation, the LC3B-I protein localized in the cytoplasm is cleaved, lapidated, and inserted as LC3BII into autophagosome membranes, ${ }^{(13)}$ while Beclin-1 prohibited these process. To detect the expression of LC3B-II and ATG5, Western blot analysis was performed with the lysates from mocktransfected and Smad7-overexpressed RGM1 cells subjected to indomethacin treatment. As seen in Fig. 1D, with indomethacin administration, the levels of LC3B-II and ATG5 were significantly increased in mock-transfected cells, while LC3B-II and ATG5 were not significantly changed even with indomethacin administration in Smad7-overexpressed cells. Instead, Beclin-1 was significantly increased in Smad7-overexpressed cells with indomethacin administration.

Smad7 overexpression significantly ameliorated indomethacin-induced LC3B activation. To further confirm whether overexpression of $\mathrm{Smad} 7$ protected indomethacininduced increase in autophagosome formation, we examined GFP-LC3 dot formation by GFP-tagged LC3 in indomethacintreated pCMV-GFP-LC3-transfected RGM1 cells. Representative fluorescence images, shown in Fig. 2A, clearly showed that compared with mock-transfected control cells, overexpression of Smad7 significantly decreased GFP-LC3 dot formation after indomethacin administration. These confocal images were further validated with Western blot for LC3B (Fig. 2B), showing knockdown of Smad7 by siRNA in RGM1 cells significantly restored indomethacin-induced LC3B-II formation $(p<0.01)$.

Mitigated indomethacin-induced cytotoxicity in Smad7overexpressed cells was mediated through p38 MAPK activation. To explore mitogen-activated protein kinases (MAPKs) contributed to rescuing action from indomethacin-induced cytotoxicity, we screened MAPK kinases, p38 and ERK1/2 between mock-transfected and Smad7-overexpressed RGM1 cells after different times, with indomethacin treatment. As seen in Fig. 3A, p38 activation was significantly noted in Smad7-overexpressed cells, remarkable increase in p38 activation after $16 \mathrm{~h}$. Though 

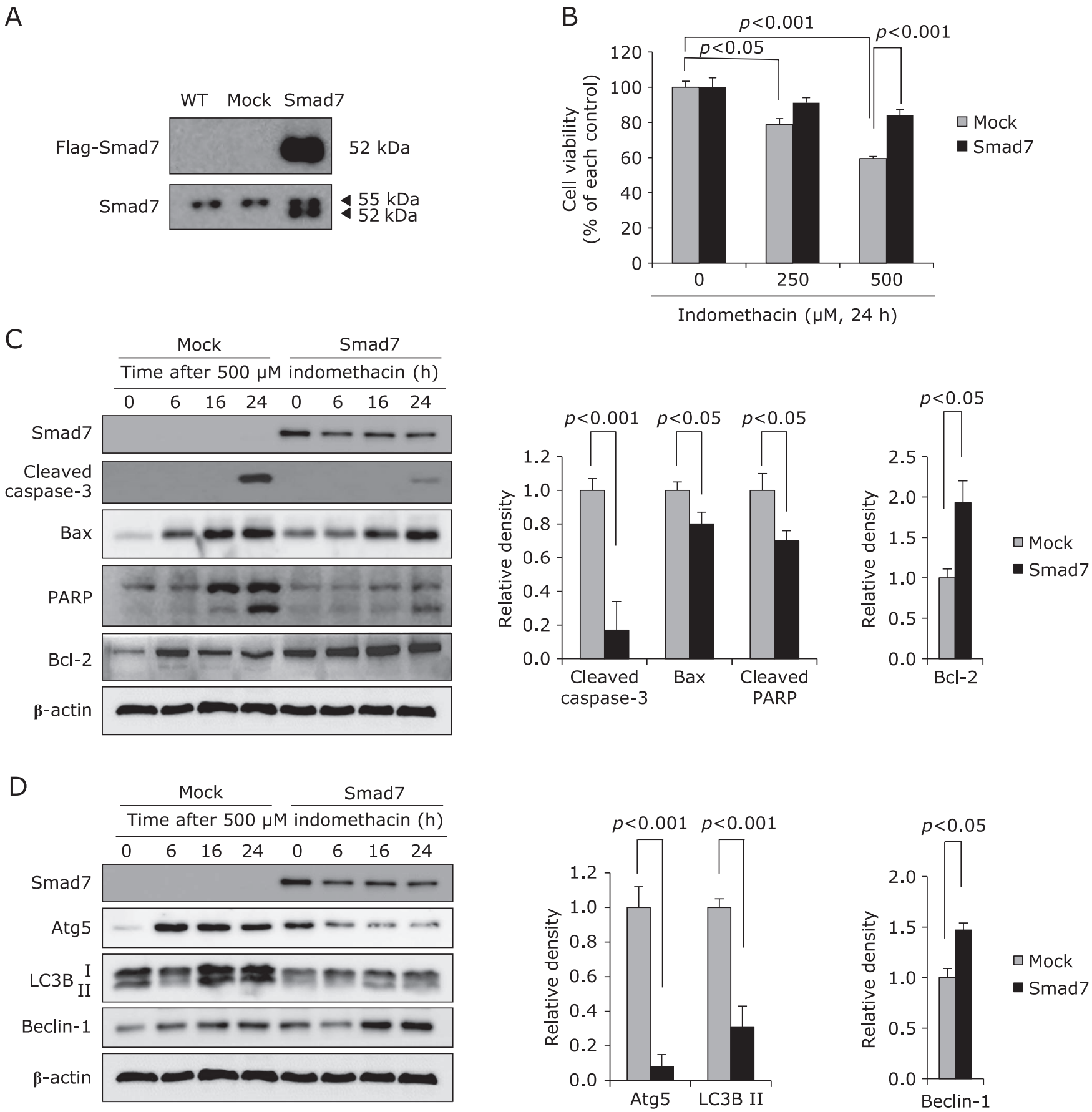

Fig. 1. Expression of Smad7 protects indomethacin-induced apoptotic and autophagic cell death in RGM1 cells. (A) Stable expression of Smad7 in RGM-1 cells was detected with Flag-tag (upper) and Smad7 (lower) antibodies. (B) RGM1 cells treated with different concentrations of indomethacin for $24 \mathrm{~h}$ were subjected to MTT assay. Bars represent mean \pm SD $(n=3)$. (C) RGM1 cells were treated with $500 \mu \mathrm{M}$ of indomethacin for different time intervals $(6-24 \mathrm{~h}$ ) and the expression of apoptotic proteins Bax, cleaved caspase-3, PARP and Bcl-2 were detected by Western blotting. (D) RGM1 cells were treated with $500 \mu \mathrm{M}$ of indomethacin for different time intervals $(6-24 \mathrm{~h})$ and the expression of autophagic markers LC3B, Beclin-1 and ATG5 were detected by Western blotting.

phosphorylated ERK was significantly decreased after indomethacin administration, while levels of ERK phosphorylation were significantly increased in Smad7-overexpressed cells, no significant changes were noted with an MEK1 inhibitor (PD98029) (Fig. 3C). The fact that TGF- $\beta$ signaling pathway activates autophagy in hepatocellular carcinoma cells, while the suppression of receptoractivated Smads abolishes the induction of autophagy significantly suggested that Smad signaling pathways are critically involved in autophagy induction. ${ }^{(14)}$ In addition, the findings that Smad7 is required for activation of p38 and overexpression of Smad7 has been demonstrated to causes activation of p38 MAPK and apoptosis in prostate cancer cells revealed that different roles of Smad7 in the regulation of the MAPK pathways and apoptosis. ${ }^{(15)}$ Taken together with the finding from Fig. $3 \mathrm{~A}$, in order to investigate whether p38 activation is relevant to rescuing action of Smad7 against indomethacin-induced autophagic cell 

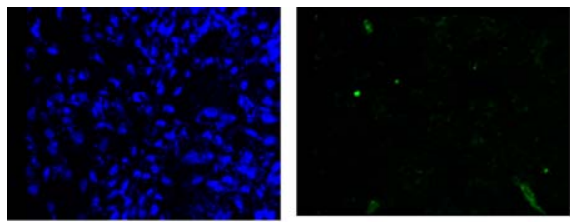

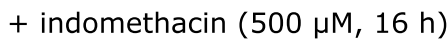

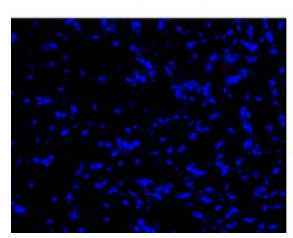

DAPI

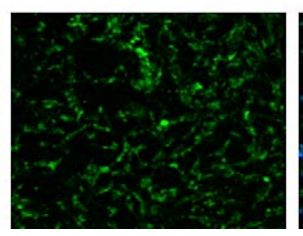

GFP-LC3B
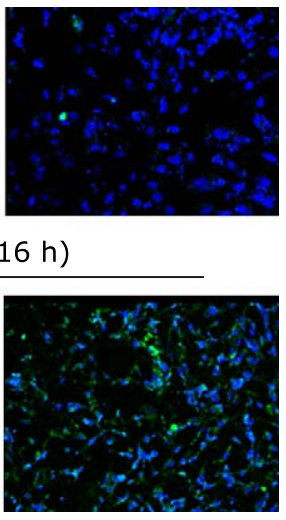

Merge

\section{RGM1-Smad7}
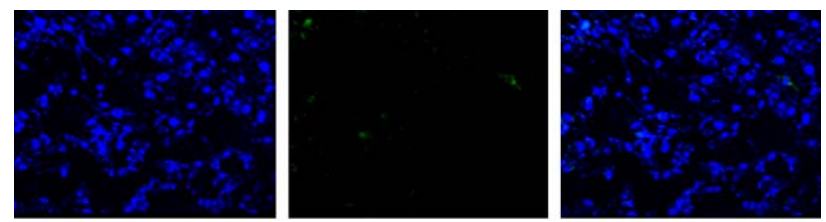

+ indomethacin (500 $\mu \mathrm{M}, 16$ h)

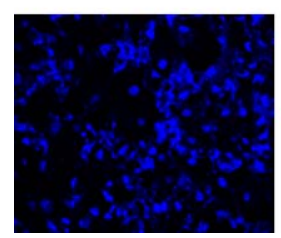

DAPI

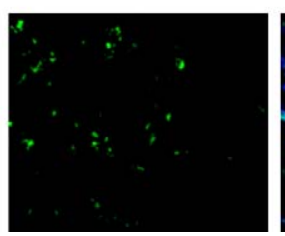

GFP-LC3B

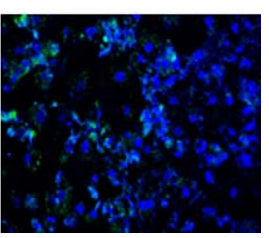

Merge
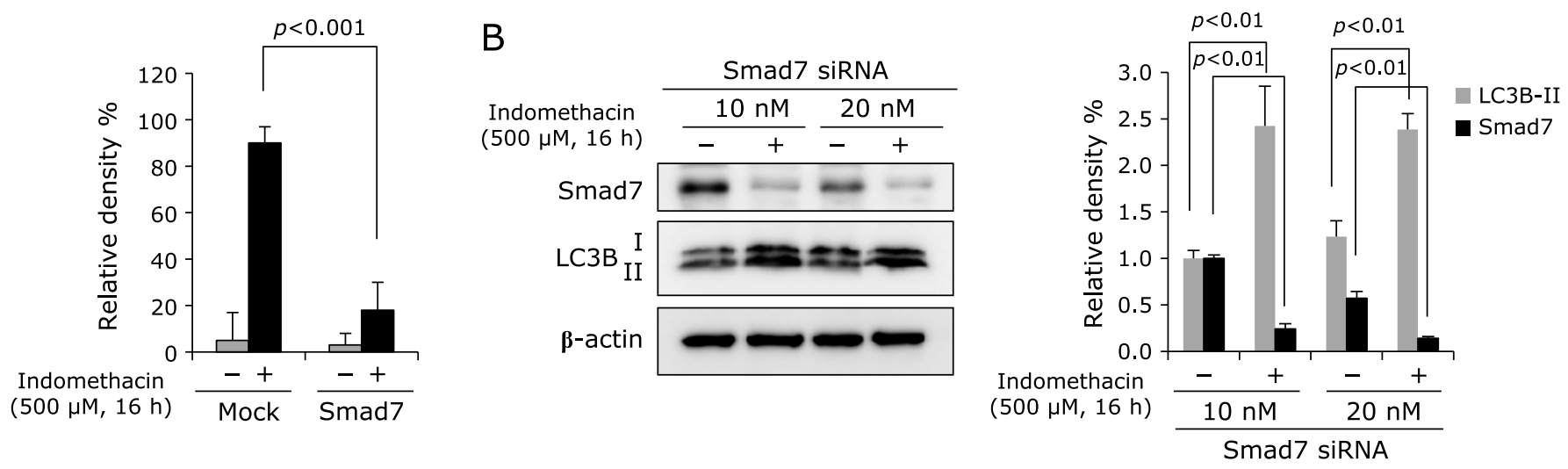

Fig. 2. Autophagy after indomethacin in mock-transfected and Smad7-overexpressed cells. (A) Smad7 expressing (RGM1-Smad7) or control (RGM1-Mock) cells transfected with GFP-LC3 was treated with $500 \mu \mathrm{M}$ of indomethacin for $16 \mathrm{~h}$. The translocation of GFP-LC3 from the cytosolic fraction to autophagic vacuoles was observed under a confocal microscope. (B) Smad7-expressed RGM1 cells transfected with different concentrations of Smad7 siRNA were treated with $500 \mu \mathrm{M}$ indomethacin for $16 \mathrm{~h}$, and cell lysates were immunoblotted with anti-Smad7, anti-LC3B and anti$\beta$-actin antibodies (left). The expression levels of Smad7 and LC3B were quantified by densitometry. Bar represents mean \pm SD, triplicate (right).

death, we determined the levels of $\mathrm{p} 38$ and LC3B in the presence p38 inhibitor, SB203580. As shown in Fig. 3B, indomethacin at $500 \mu \mathrm{M}$ for $16 \mathrm{~h}$ significantly decreased the levels of Smad7 compared to before indomethacin administration, while in this condition, p38 activation was significantly increased. In mocktransfected cells, indomethacin administration significantly increased LC3B-II after indomethacin administration, but no change in Smad7-overexpressed cells, suggesting LC3B activation after indomethacin was reversely influenced by $\mathrm{p} 38$ activation. These findings were further confirmed by using a p38 inhibitor, as seen in Fig. 3B, SB203580 together with indomethacin administration in Smad7-overexpressed cells showed LC3B activation, increases in LC3B-II expressions compared with indomethacin administration in smad7-overexpressed cells. However, no significant changes were noted in Smad7-overexpressed cells irrespective of MEK-1 inhibitor treatment (Fig. 3C). Generally, indomethacin was reported to increase COX-2 expression. ${ }^{(16)}$ As seen in Fig. 3D, indomethacin administration significantly increased COX-2 expression in mock-transfected cells and Smad7-overexpressed cells. However, the p38 inhibitor significantly decreased COX-2 expression in mock-transfected cells, while no significant changes were noted in Smad7-overexpressed cells in the presence of p38 inhibitor, suggesting biological changes in Smad7-overexpressed cells after indomethacin administration were influenced by p38 activation.
Indomethacin degraded Smad7 through the ubiquitinproteasome pathway. During investigation, we have found significant decrements in Smad7 expression in Smad7overexpressed cells after indomethacin administration (Fig. 1C, $1 \mathrm{D}$ and 3B). Consistently, data showed the possibility of Smad7 degradation or expression inhibition with indomethacin. To see whether indomethacin affects the expression of Smad7, we repeated the analysis of the mRNA expression of Smad7 in control and Smad7-overexpressed RGM1 cells treated with $500 \mu \mathrm{M}$ of indomethacin. As noted in Fig. 4A, indomethacin slightly decreased Smad7 mRNA in Smad7-overexpressed RGM1 cells, while it had no effect in control cells. However, as shown in Fig. 4B, indomethacin administration significantly decreased the expression of Smad7 dependent on exposure time, making the hypothesis that indomethacin might degrade $\mathrm{Smad} 7$ through post-transcriptional regulation. Therefore, in order to document whether decrease of Smad7 protein levels after indomethacin is result from increased ubiquitination, we have measured ubiquitinconjugated proteins after indomethacin treatment in Smad7overexpressed RGM1 cells. As shown in Fig. 4C, indomethacin treatment resulted in a significant increase in the ubiquitination of Smad7 as well as total ubiquitinated proteins, whereas proteasome inhibitor MG132 blocked ubiquitin-mediated degradation of Smad7 (Fig. 3D). Taken together with the above experimental results that in the absence of Smad7 (siSmad7 transfection) 
A

$\frac{\text { Mock }}{\text { Time after indomethacin } 500 \mu \mathrm{M}(\mathrm{h})}$

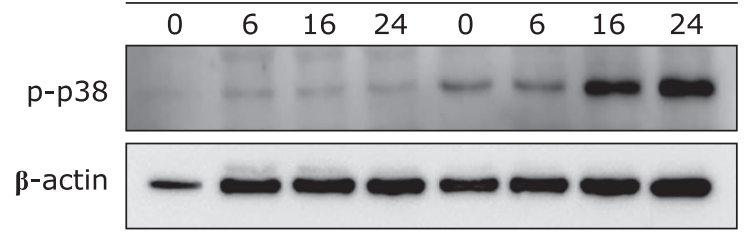

C
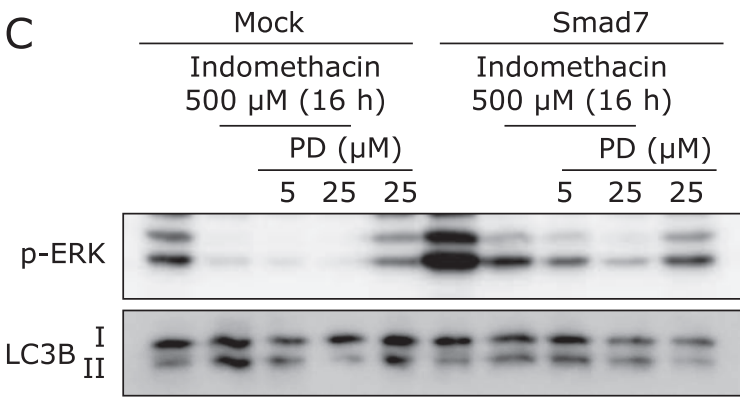

$\beta$-actin

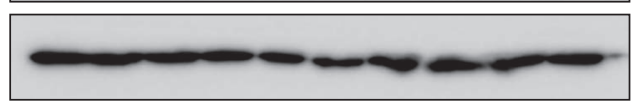

D
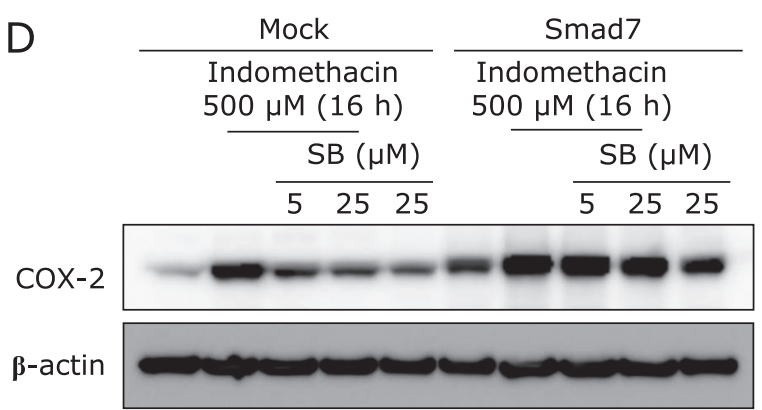

B
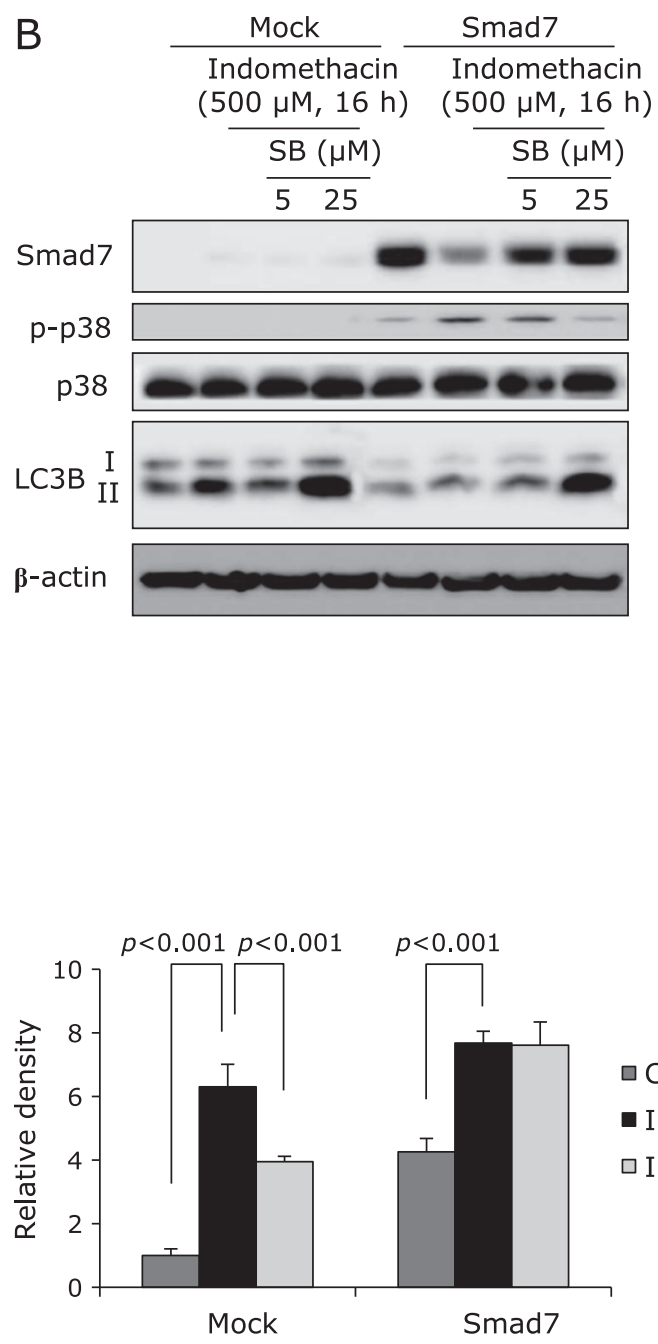

$\square$ Control

- Indomethacin

$\square$ Indomethacin + SB

Fig. 3. p38 MAPK plays an important role in indomethacin-induced autophagic cell death. (A) Smad7 expressing (RGM1-Smad7) or control (RGM1Mock) cells were treated with $500 \mu \mathrm{M}$ of indomethacin for different time intervals $(6-24 \mathrm{~h})$ and the activation of p38 was detected by Western blotting. (B) Western blot of phospho-p38 and LC3B after treatment of SB203580 and indomethacin in mock-transfected or Smad7-expressed RGM1 cells. (C) Western blot of phospho-ERK and LC3B after treatment of PD98029 and indomethacin in control or Smad7 expressing RGM1 cells. (D) Western blot of COX-2 after after treatment of SB203580 and indomethacin in mock-transfected or Smad7-expressed RGM1 cells. The ratio of COX-2 to $\beta$-actin was quantified by densitometry. Bar represents mean \pm SD, triplicate (right).

indomethacin significantly increased LC3B-II (Fig. 2B) and further LC3B activation with MG132 under Smad7 siRNA (Fig. 4E) commonly implied significant and critical implication of Smad7 in NSAID-induced autophagic cell death, Smad7 degradation after indomethacin add cytotoxicity pathway.

Chloroquine as Smad7 inducer mitigated indomethacininduced cell death in RGM1 cells. On the basis of previous findings that overexpression or preservation of Smad7 protein in gastric epithelial cells alleviated indomethacin-induced gastric epithelial cytotoxicity, we searched pharmacological inducers of Smad7. During screening agents, we found that CQ significantly induces mRNA and protein levels of Smad7 in RGM1 cells (Fig. 5A). CQ, an old antimalarial drug, is a highly promising autophagy inhibitor for clinical use $\mathrm{u}^{(17,18)}$ against rheumatoid arthritis and possibility of direct anti-cancer or chemotherapy enhancing action. ${ }^{(19-21)}$ Therefore, we continued to prove what happened when pharmacological induction of Smad7 with CQ was imposed to indomethacin-associated cytotoxicity. As seen in Fig. 5B and 5C, indomethacin significantly increased apoptosis as measured by Annexin V and cleaved caspase-3 ( $p<0.01)$, but co- treatment with $6 \mu \mathrm{M} \mathrm{CQ}$ and $500 \mu \mathrm{M}$ indomethacin significantly decreased cell apoptosis $(p<0.01)$. These results suggest that Smad7 played protection from indomethacin-induced cytotoxicity and pharmacological inducers of Smad7 can rescue stomach from indomethacin-induced gastric damages.

\section{Discussion}

In the current study, for the first time, it was clearly documented that NSAID-induced apoptotic or autophagic cell death was accompanied with Smad7 depletion. The explored fact that degradation of Smad7 as COX-independent cytotoxic mechanisms responsible for NSAID-induced gastric epithelial cell injury led to anticipation that autophagy inhibition or Smad7 preservation can be an anticipating strategy rescuing from NSAID-induced gastric damages (Fig. 6). Though selective COX-2 inhibitor (coxibs) or combination of NSAID and proton pump inhibitor (PPI) is preferred strategy to mitigate NSAID-induced cytotoxicity, several limitations that risk of thromboembolic events, the leaky gut syndrome or collagenous colitis after PPI combina- 

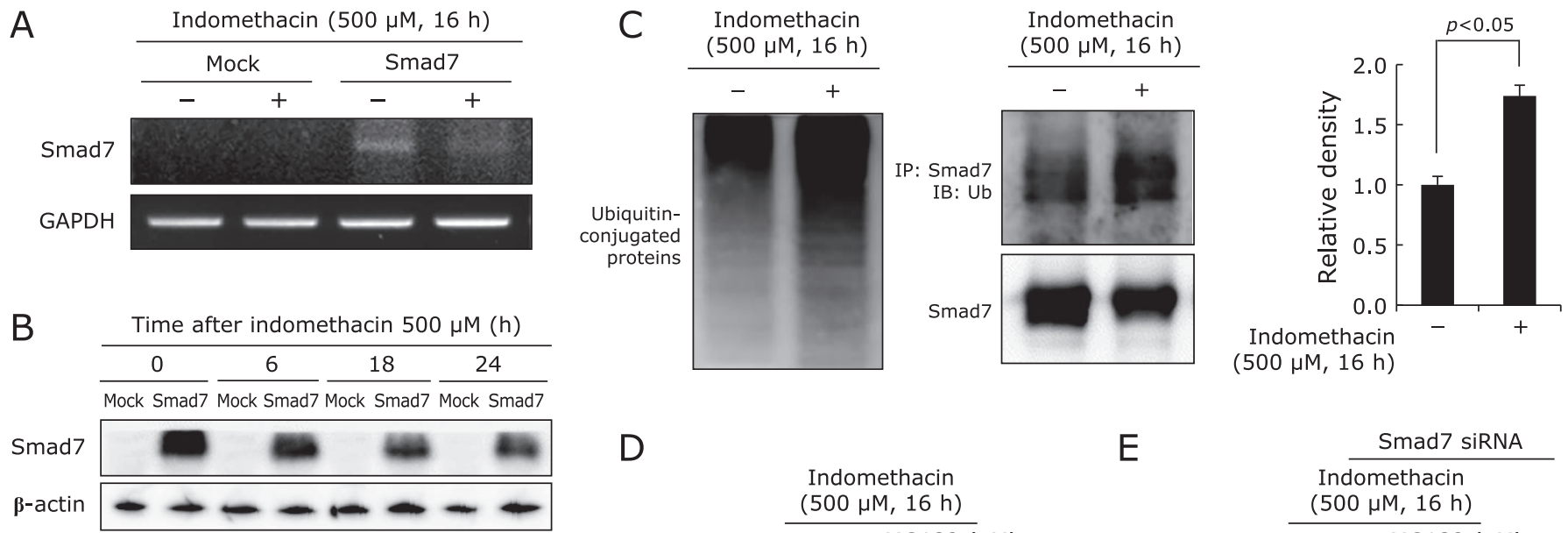

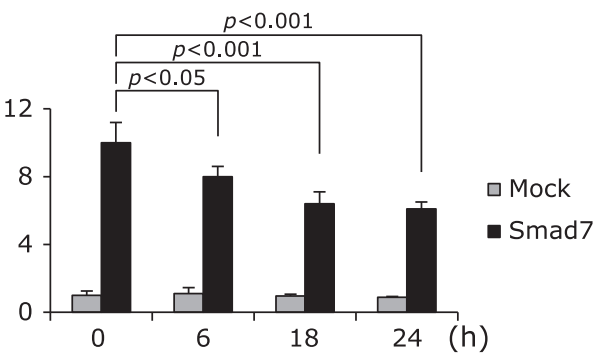

D

Indomethacin (500 $\mu \mathrm{M}, 16 \mathrm{~h}$ )

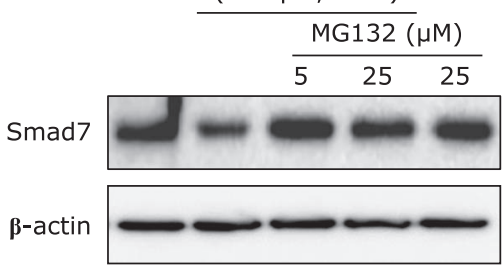

$\mathrm{E}$
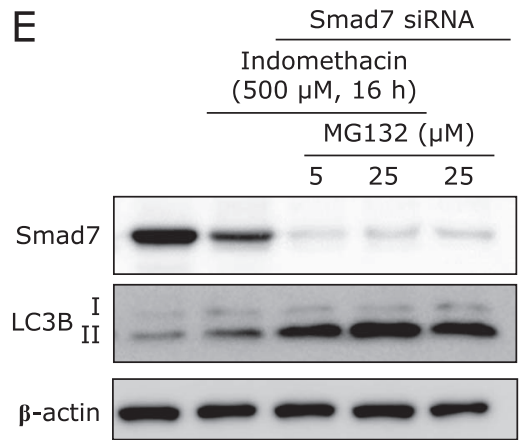

$\beta$-actin (500 $\mu \mathrm{M}, 16 \mathrm{~h}$ )

Fig. 4. Indomethacin treatment induces proteasome-dependent degradation of Smad7 in RGM1 cells. Smad7 expressing (RGM1-Smad7) or control (RGM1-Mock) cells were treated with $500 \mu \mathrm{M}$ of indomethacin and the mRNA expression (A) and protein levels of Smad7 (B) were detected by RT-PCR and Western blotting, respectively. (C) Smad7-expressed RGM1 cells were treated with $500 \mu \mathrm{M}$ of indomethacin for $16 \mathrm{~h}$ and the protein lysates were immunoprecipitated with anti-Smad7 antibody and immunoblotted with anti-ubiquitin antibody. (D) Smad7-expressed RGM1 cells were treated with $500 \mu \mathrm{M}$ of indomethacin for $16 \mathrm{~h}$ in the presence of absence of MG132 and protein levels of Smad7 were detected by Western blotting. (E) Smad7-expressed RGM1 cells were treated with $500 \mu \mathrm{M}$ of indomethacin for $16 \mathrm{~h}$ in the presence of absence of Smad7 siRNA and the protein levels of Smad7 and LC3B were detected by Western blotting.

tion, increased intestinal permeability the risk of small intestinal damages, and direct toxicity of NSAIDs still threatened the NSAID use in elderly person. In this point of view, out study opens the possibility looking at rescuing from NSAID-induced gastroenteropathy with the novel strategy of Smad7 preservation. From our study, CQ or other compounds enhancing Smad7 expression achieved equivalent or higher efficacy against indomethacininduced gastric damages than current coxibs or PPI combination, but the necessity of well-designed clinical trials for future clinical application is needed.

In the current study, quite novel findings regarding the protection from NSAID cytotoxicity were explored onto the following two matters, one was regarding Smad7 preservation and the other was CQ revisited as autophagy inhibitor as well as Smad7 inducer. Recently great achievement was done relevant to Smad7 anti-sense oligonucleotide in the treatment of inflammatory bowel disease, where the restoration of anti-inflammatory cytokine and TGF- $\beta$ signaling through Smad7 inhibition. ${ }^{(22)}$ In this condition, TGF- $\beta$ inhibitory Smad7 was implicated in the propagation of intestinal inflammation, after which anti-sense oligonucleotide, Mongersen, effectively restored anti-inflammatory role of TGF- $\beta$. In the current study, Smad7 contributed to limit the cytotoxic influence of NSAIDs, by which CQ as Smad7 inducer significantly contributed to rescuing action of NSAID cytotoxicity.

It has been demonstrated that Smad7 can interact with other intracellular proteins and regulate also TGF- $\beta$-independent signaling pathways, should be cautiously interpreted according to disease condition. For instance, Smad6 or Smad7, even though they are inhibitory mediators of anti-inflammatory TGF- $\beta$, contributed to ameliorate allergic dermatitis, radiation stomatitis, or sepsis. ${ }^{(23)}$ Different with the above inflammatory bowel disease or Helicobacter pylori-associated gastritis, Smad7 exerted overt protective action against NSAID-induced cytotoxicity in the current study. Though CQ has been used for malaria for over 500 years as well as disease-modifying properties in systemic lupus erythematosus for over 50 years, ${ }^{(24)}$ newer iterations of this class of anti-inflammatory agents was done on the treatment of autoimmune diseases as well as indication as for inflammatory diseases based on Toll-like receptor-associated mechanisms. In the current study, we put forward as potential autophagy inhibitor against NSAID-associated cytotoxicity. Some researchers put CQ into an anticancer drug as new horizons for old drugs, ${ }^{(17,18)}$ in this indication as autophagy inhibitor targeting cancer metabolism. Clinical trials are ongoing where CQ or hydroxyl CQ are used to sensitizes cancer cells to chemotherapy independent of autophagy and where repositioning CQ to eliminate cancer stem cells in premalignant lesions. ${ }^{(25,26)}$ Our study might be the first to target CQ as for relieving NSAID-induced gastric damages focused onto the specific mechanisms of NSAID cytotoxicity.

Lastly, ever novel finding relevant to Smad7 expression after NSAID was NSAID led to Smad7 degradation via ubiquitinproteasome pathway. As seen in Fig. 4, indomethacin led to significant repressed status of Smad7, rapid down-regulation of Smad7 consistent with its accelerated degradation. In the literature, aristolochic acid-induced nephropathy and streptozotocininduced diabetic nephropathy were reported with significant ubiquitination of Smad7, ${ }^{27,28)}$ but never in NSAID-induced GI damages before our investigation. Though not investigated in the 
A
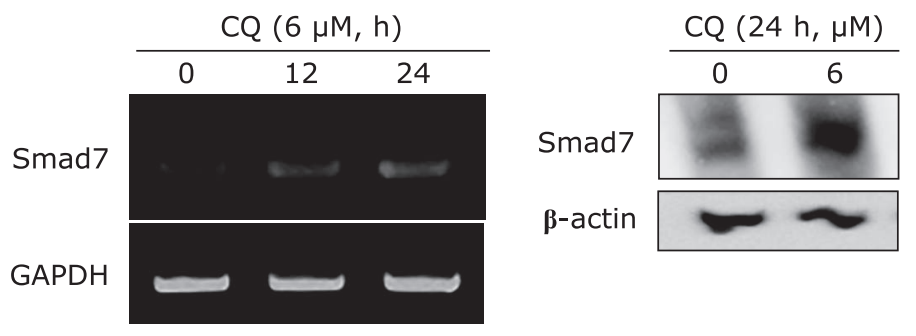

C

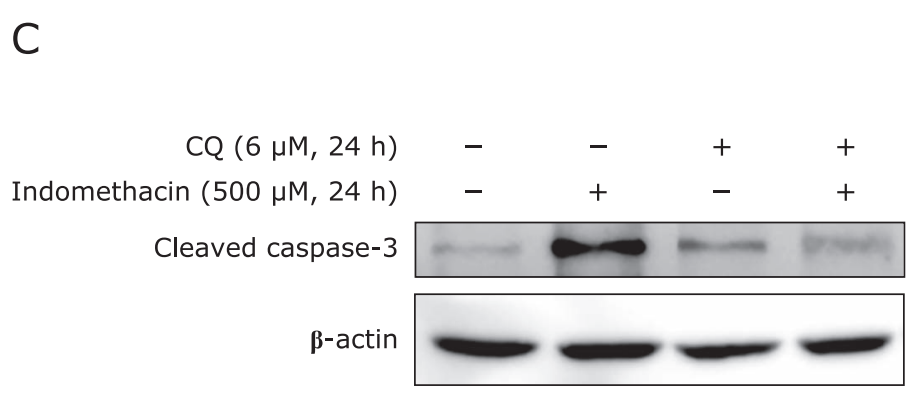

B

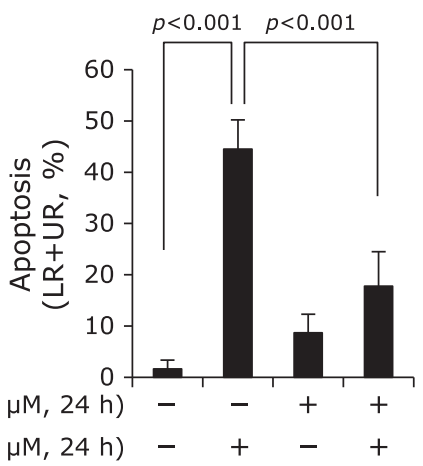

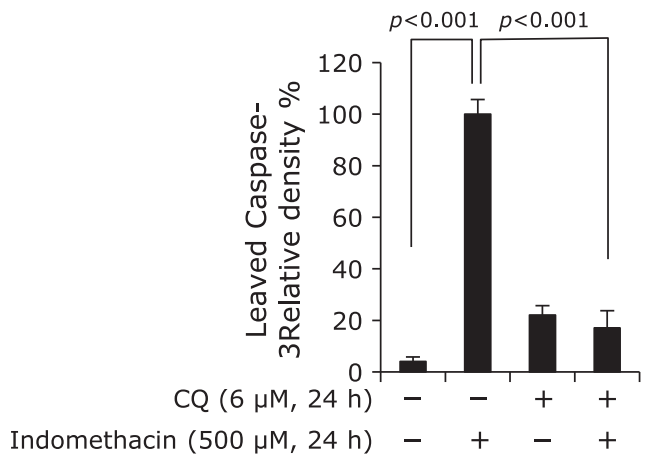

Fig. 5. Chloroquine rescues indomethacin-induced apoptotic cell death in RGM1 cells. (A) RGM1 cells were treated with chloroquine, and the mRNA expression and protein levels of Smad7 were detected by RT-PCR and Western blotting, respectively. RGM1 cells were treated with $500 \mu \mathrm{M}$ of indomethacin for $16 \mathrm{~h}$ in the presence or absence of chloroquine, the levels of apoptosis (B) and cleaved caspase-3 (C) were detected by flow cytometry and Western blotting, respectively. The ratio of cleaved caspase- 3 to $\beta$-actin was quantified by densitometry. Bars represent mean \pm SD $(n=3)$.

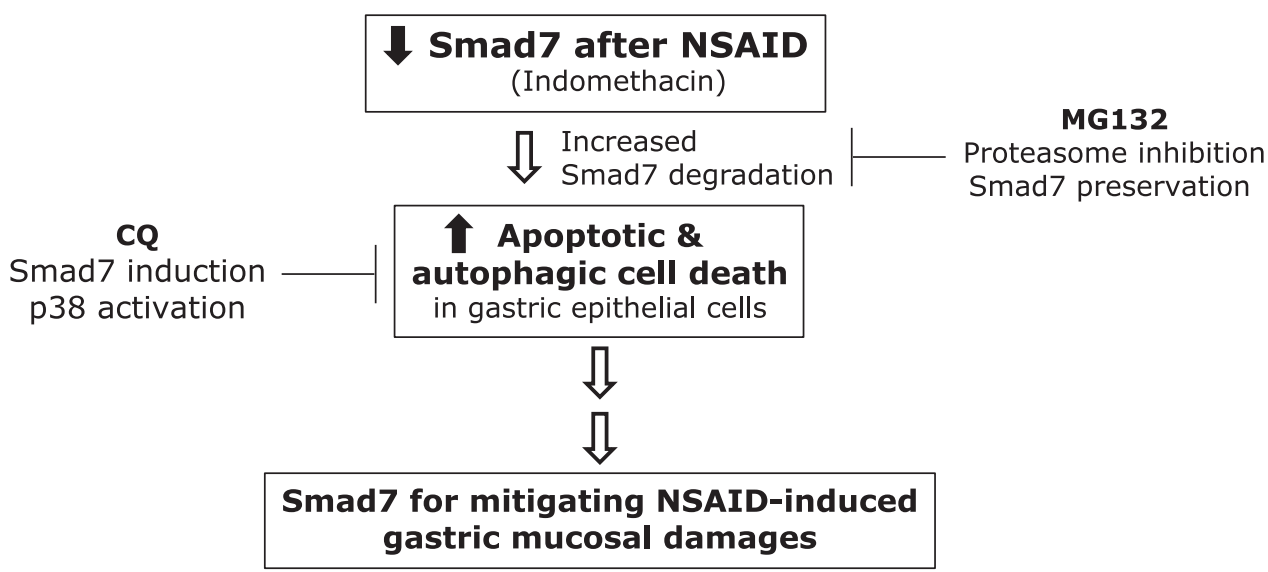

Fig. 6. Preservation of Smad7 expression rescues NSAID-induced cytotoxicity. Schematic summary to explain Smad7 preservation can be an anticipating strategy rescuing from NSAID-induced gastric damages.

current study further, an important regulatory step involving specific ubiquitination by Smurfs (Smad-ubiquitin regulatory factors), members of the homologous to E6-associated protein Cterminus ubiquitin ligase family, mediates the proteasomal degradation of Smads and/or receptors. ${ }^{(29)}$ Though differentially implicated, Smad7 was also post-transcriptionally regulated in inflammatory bowel disease, its increase in patients with inflammatory bowel disease is due to stabilization by p300 acetylation, thus, preventing Smad7 ubiquitination and proteasomal degradation, that is, Smad7 protein in cells from normal gut is ubiqui- tinated, whereas in inflamed gut, Smad7 is acetylated and not ubiquitinated, is not degraded. ${ }^{(30)}$ Translating with our investigation, the levels of Smad7 in mock-transfected cells were very low, but in case of increased Smad7 levels, NSAID significantly increased proteasomal degradation, enabling susceptible to NSAIDinduced cell damages. Therefore, MG132, other natural products as well as CQ can be possible candidates for rescuing NSAIDinduced GI damages after further investigations.

Since NSAID-induced apoptosis or autophagy seems to be allied to license cell death, optimal modulation of autophagy can 
be an earnest opportunity to attenuate NSAID-associated gastric damages. However, extensive large scale of clinical study will be required to put autophagy inhibitor as novel therapeutic strategy to rescue stomach from NSAID-induced gastric damages.

\section{Acknowledgments}

This research was supported by Gachon University Gil Medical Center research fund FRD2013-17 (Lee HJ), and by the Basic Science Research Program through the National Research Foun-

\section{References}

1 Graham DY, Chan FK. NSAIDs, risks, and gastroprotective strategies: current status and future. Gastroenterology 2008; 134: 1240-1246.

2 Handa O, Naito Y, Fukui A, Omatsu T, Yoshikawa T. The impact of nonsteroidal anti-inflammatory drugs on the small intestinal epithelium. $J$ Clin Biochem Nutri 2014; 54: 2-6.

3 Vane JR. Inhibition of prostaglandin synthesis as a mechanism of action for aspirin-like drugs. Nat New Biol 1971; 231: 232-235.

4 Saito R, Tamua M, Matsui H, et al. Qing Dai attenuates nonsteroidal antiinflammatory drug-induced mitochondrial reactive species in gastrointestinal epithelial cells. J Clin Biochem Nutri 2015; 56: 8-14.

5 Tsutsumi S, Gotoh T, Tomisato W, et al. Endoplasmic reticulum stress response is involved in nonsteroidal anti-inflammatory drug-induced apoptosis. Cell Death Differ 2004; 11: 1009-1016.

6 Chen N, Karantza V. Autophagy as a therapeutic target in cancer. Cancer Biol Ther 2011; 11: 157-168.

7 Chen HY, White E. Role of autophagy in cancer prevention. Cancer Prev Res (Phila) 2011; 4: 973-983.

8 Marciniak SJ, Ron D. Endoplasmic reticulum stress signaling in disease. Physiol Rev 2006; 86: 1133-1149.

9 Ogata M, Hino S, Saito A, et al. Autophagy is activated for cell survival after endoplasmic reticulum stress. Mol Cell Biol 2006; 26: 9220-9231.

10 Kouroku Y, Fujita E, Tanida I, et al. ER stress (PERK/eIF2alpha phosphorylation) mediates the polyglutamine-induced LC3 conversion, an essential step for autophagy formation. Cell Death Differ 2007; 14: 230-239.

11 Li J, Hou N, Faried A, Tsutsumi S, Takeuchi T, Kuwano H. Inhibition of autophagy by 3-MA enhances the effect of 5-FU-induced apoptosis in colon cancer cells. Ann Surg Oncol 2009; 16: 761-771.

12 Li J, Hou N, Faried A, Tsutsumi S, Kuwano H. Inhibition of autophagy augments 5-fluorouracil chemotherapy in human colon cancer in vitro and in vivo model. Eur J Cancer 2010; 46: 1900-1909.

13 Wild P, McEwan DG, Dikic I. The LC3 interactome at a glance. J Cell Sci 2014; 127 (Pt 1): 3-9.

14 Kiyono K, Suzuki HI, Matsuyama H, et al. Autophagy is activated by TGFbeta and potentiates TGF-beta-mediated growth inhibition in human hepatocellular carcinoma cells. Cancer Res 2009; 69: 8844-8852.

15 Edlund S, Bu S, Schuster N, et al. Transforming growth factor-beta1 (TGFbeta)-induced apoptosis of prostate cancer cells involves Smad7-dependent activation of $\mathrm{p} 38$ by TGF-beta-activated kinase 1 and mitogen-activated protein kinase kinase 3. Mol Biol Cell 2003; 14: 529-544.

16 Tanaka A, Hase S, Miyazawa T, Takeuchi K. Up-regulation of cyclooxygenase-2 by inhibition of cyclooxygenase-1: a key to nonsteroidal dation of Korea (NRF) funded by the Ministry of Education (2014R1A1A2058732), by Korea Institute of Planning and Evaluation for Technology in Food, Agriculture, Forestry and Fisheries (IPET) through High Value-added Food Technology Development Program, funded by Ministry of Agriculture, Food and Rural Affairs (MAFRA) (116015-03-1-CG000).

\section{Conflict of Interest}

No potential conflicts of interest were disclosed.

anti-inflammatory drug-induced intestinal damage. J Pharmacol Exp Ther 2002; 300: 754-761.

17 Yang ZJ, Chee CE, Huang S, Sinicrope FA. The role of autophagy in cancer: therapeutic implications. Mol Cancer Ther 2011; 10: 1533-1541.

18 Amaravadi RK, Lippincott-Schwartz J, Yin XM, et al. Principles and current strategies for targeting autophagy for cancer treatment. Clin Cancer Res 2011; 17: 654-666.

19 Augustijns P, Geusens P, Verbeke N. Chloroquine levels in blood during chronic treatment of patients with rheumatoid arthritis. Eur J Clin Pharmacol 1992; 42: 429-433.

20 Kimura T, Takabatake Y, Takahashi A, Isaka Y. Chloroquine in cancer therapy: a double-edged sword of autophagy. Cancer Res 2013; 73: 3-7.

21 Pascolo S. Time to use a dose of Chloroquine as an adjuvant to anti-cancer chemotherapies. Eur J Pharmacol 2016; 771: 139-144.

22 Monteleone G, Neurath MF, Ardizzone S, et al. Mongersen, an oral SMAD7 antisense oligonucleotide, and Crohn's disease. $N$ Engl J Med 2015; 372: 1104-1113.

23 Han G, Bian L, Li F, et al. Preventive and therapeutic effects of Smad7 on radiation-induced oral mucositis. Nat Med 2013; 19: 421-428.

24 Wallace DJ, Gudsoorkar VS, Weisman MH, Venuturupalli SR. New insights into mechanisms of therapeutic effects of antimalarial agents in SLE. Nat Rev Rheumatol 2012; 8: 522-533.

25 Maycotte P, Aryal S, Cummings CT, Thorburn J, Morgan MJ, Thorburn A. Chloroquine sensitizes breast cancer cells to chemotherapy independent of autophagy. Autophagy 2012; 8: 200-212.

26 Vazquez-Martin A, López-Bonetc E, Cufí S, et al. Repositioning chloroquine and metformin to eliminate cancer stem cell traits in pre-malignant lesions. Drug Resist Updat 2011; 14: 212-223.

27 Tian Y, Liao F, Wu G, et al. Ubiquitination and regulation of Smad7 in the TGF- $\beta 1 /$ Smad signaling of aristolochic acid nephropathy. Toxicol Mech Methods 2015; 25: 645-652.

28 Gao C, Aqie K, Zhu J, et al. MG132 ameliorates kidney lesions by inhibiting the degradation of Smad7 in streptozotocin-induced diabetic nephropathy. $J$ Diabetes Res 2014; 2014: 918396.

29 Wicks SJ, Grocott T, Haros K, Maillard M, ten Dijke P, Chantry A Reversible ubiquitination regulates the Smad/TGF-beta signalling pathway. Biochem Soc Trans 2006; 34 (Pt 5): 761-763.

30 Monteleone G, Del Vecchio Blanco G, Monteleone I, et al. Post-transcriptional regulation of $\mathrm{Smad} 7$ in the gut of patients with inflammatory bowel disease. Gastroenterology 2005; 129: 1420-1429. 